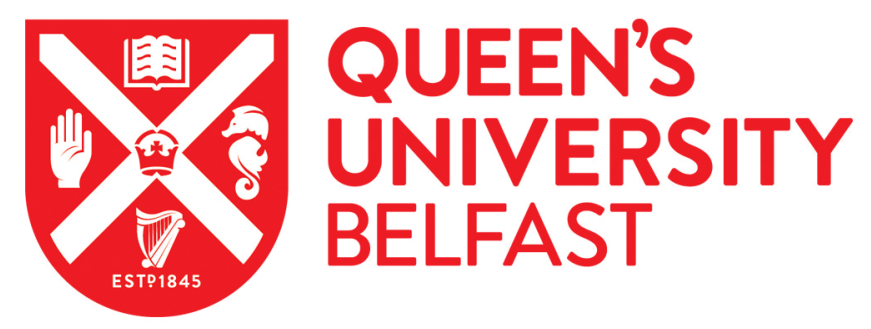

\title{
Measurement of the full stress tensor in a crystal using photoluminescence from point defects: The example of nitrogen vacancy centers in diamond
}

Grazioso, F., Patton, B. R., Delaney, P., Markham, M. L., Twitchen, D. J., \& Smith, J. M. (2013). Measurement of the full stress tensor in a crystal using photoluminescence from point defects: The example of nitrogen vacancy centers in diamond. Applied Physics Letters, 103(10), [101905]. https://doi.org/10.1063/1.4819834

Published in:

Applied Physics Letters

Document Version:

Publisher's PDF, also known as Version of record

Queen's University Belfast - Research Portal:

Link to publication record in Queen's University Belfast Research Portal

\section{Publisher rights}

Copyright 2013 American Institute of Physics. This article may be downloaded for personal use only. Any other use requires prior permission of the author and the American Institute of Physics.

\section{General rights}

Copyright for the publications made accessible via the Queen's University Belfast Research Portal is retained by the author(s) and / or other copyright owners and it is a condition of accessing these publications that users recognise and abide by the legal requirements associated with these rights.

Take down policy

The Research Portal is Queen's institutional repository that provides access to Queen's research output. Every effort has been made to ensure that content in the Research Portal does not infringe any person's rights, or applicable UK laws. If you discover content in the

Research Portal that you believe breaches copyright or violates any law, please contact openaccess@qub.ac.uk. 


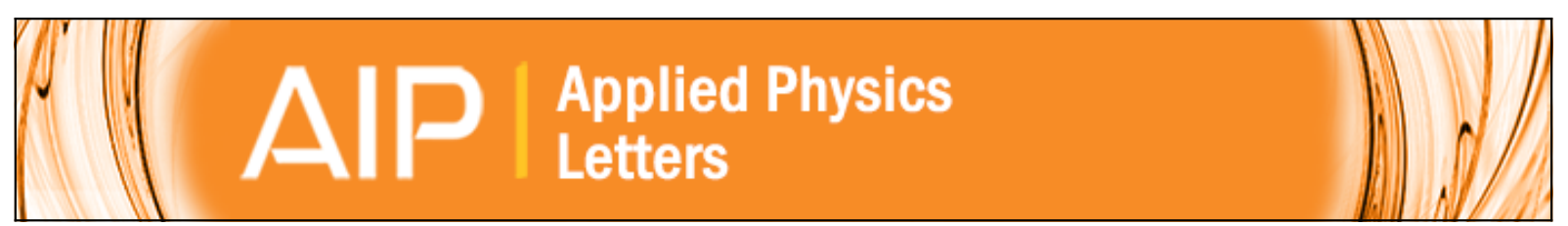

Measurement of the full stress tensor in a crystal using photoluminescence from point defects: The example of nitrogen vacancy centers in diamond

Fabio Grazioso, Brian R. Patton, Paul Delaney, Matthew L. Markham, Daniel J. Twitchen, and Jason M. Smith

Citation: Applied Physics Letters 103, 101905 (2013); doi: 10.1063/1.4819834

View online: http://dx.doi.org/10.1063/1.4819834

View Table of Contents: http://scitation.aip.org/content/aip/journal/apl/103/10?ver=pdfcov

Published by the AIP Publishing

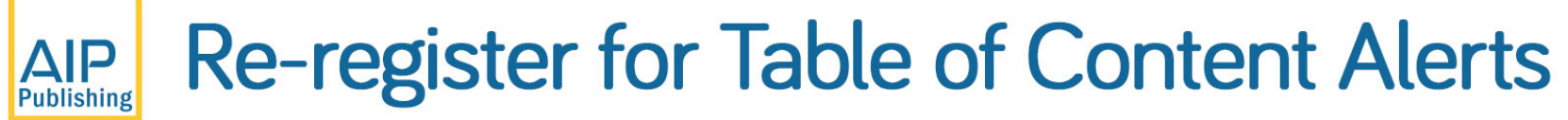




\title{
Measurement of the full stress tensor in a crystal using photoluminescence from point defects: The example of nitrogen vacancy centers in diamond
}

\author{
Fabio Grazioso, ${ }^{1}$ Brian R. Patton, ${ }^{1}$ Paul Delaney, ${ }^{2, a)}$ Matthew L. Markham, ${ }^{3}$ \\ Daniel J. Twitchen, ${ }^{3}$ and Jason M. Smith ${ }^{1, b)}$ \\ ${ }^{1}$ Department of Materials, University of Oxford, Parks Road, Oxford OX1 3PH, United Kingdom \\ ${ }^{2}$ School of Mathematics and Physics, Queen's University Belfast, Belfast BT7 1NN, Northern Ireland \\ ${ }^{3}$ Element Six Ltd., King's Ride Park, Ascot, Berkshire SL5 8BP, United Kingdom
}

(Received 22 May 2013; accepted 24 July 2013; published online 6 September 2013)

\begin{abstract}
We introduce a method for measuring the full stress tensor in a crystal utilising the properties of individual point defects. By measuring the perturbation to the electronic states of three point defects with $C_{3 v}$ symmetry in a cubic crystal, sufficient information is obtained to construct all six independent components of the symmetric stress tensor. We demonstrate the method using photoluminescence from nitrogen-vacancy colour centers in diamond. The method breaks the inverse relationship between spatial resolution and sensitivity that is inherent to existing bulk strain measurement techniques, and thus, offers a route to nanoscale strain mapping in diamond and other materials in which individual point defects can be interrogated. (C) 2013 AIP Publishing LLC. [http://dx.doi.org/10.1063/1.4819834]
\end{abstract}

Since the proposal by Kane for a quantum computer based on phosphorus impurities in silicon, ${ }^{1}$ considerable effort has focused on developing solid state quantum spintronic devices involving individually addressable qubits in crystalline materials. The properties of these solid state qubits depend significantly on the local crystal environment such as lattice strain and the presence of extraneous impurities and defects, and so the ability to probe these features will be essential in developing robust devices. However, measuring stress and strain distributions on the length scales required presents a significant challenge. Existing methods for measuring strain in materials, Raman spectroscopy, ${ }^{2}$ cathodoluminescence, ${ }^{3}$ photoelasticity, ${ }^{4}$ and electron back scattering diffraction (EBSD), ${ }^{5}$ are incapable of nanoscale spatial resolution since they measure properties (phonon energies, band gaps) that depend on the translational symmetry and periodicity of the crystal lattice, and achieve high sensitivity only by averaging over a large number of lattice periods. Moreover, the fact that stress and strain are represented by second rank field tensors with, in general, six independent components, ${ }^{6}$ means that a full stress/strain map requires at least six parameters to be measured for each point in space. Only EBSD and off-axis Raman spectroscopy allow measurement of all six components of the strain tensor, and neither of these is well suited to volumetric imaging.

In this letter, we present a method for measuring the full stress tensor in diamond using the properties of point defects, namely, fluorescence from negatively charged nitrogenvacancy (NV) centers. Since the NV centers are localised effectively to single lattice sites within the crystal and possess electronic states that are sensitive to minute changes in the relative positions of the neighbouring atoms, high spatial resolution and high sensitivity are achieved simultaneously. With a suitable distribution of NV centers in the material

\footnotetext{
${ }^{a)}$ Present address: Tyndall National Institute, University College Cork, Lee Maltings, Prospect Row, Cork, Ireland.

b) jason.smith@materials.ox.ac.uk. URL: www-png.materials.ox.ac.uk
}

and using high resolution microscopy, ${ }^{7}$ the method offers a means to map the strain tensor with $\sim 10 \mathrm{~nm}$ spatial resolution, sufficient for the identification of other lattice defects such as substitutional nitrogen via their local strain fields.

NV centers in diamond provide an ideal system for this study: they are bright, stable emitters ${ }^{8}$ with well understood strain signatures in the optical spectrum. ${ }^{9-13}$ They are also leading candidates as solid state spin qubits, ${ }^{14,15}$ and with a recently reported ${ }^{13} \mathrm{C}$ spin coherence time in excess of a second ${ }^{16}$ and NV gate times of order nanoseconds, ${ }^{17}$ robust quantum processors involving arrays of $\mathrm{NV}$ centers coupled to nuclear spins are an increasingly realistic prospect. Moreover, the engineering of strain in diamond may itself be important to the functionality of NV center based devices-identifying NV centers with appropriate strain perturbations has been central to the demonstration of coherent population trapping ${ }^{18}$ and spin-photon entanglement. ${ }^{19}$

The NV defect comprises a substitutional nitrogen atom and a vacancy in an adjacent lattice site along one of the four $\langle 111\rangle$ crystal axes of the diamond lattice. The electronic structure of the color center ${ }^{9-11}$ includes a ${ }^{3} \mathrm{~A}_{2}$ orbital singlet ground state and ${ }^{3} \mathrm{E}$ orbital doublet optically excited state at $1.945 \mathrm{eV}$, the electron wave functions of which are linear superpositions of the dangling $s p^{3}$ orbitals directed in to the vacancy. ${ }^{20}$ It is this ${ }^{3} \mathrm{E} \rightarrow{ }^{3} \mathrm{~A}_{2}$ optical transition that we will use to demonstrate the stress tensor measurement.

According to the group operator replacement theorem, ${ }^{22}$ the effect of strain on an individual NV center is indistinguishable from that of a vector field, since the perturbation Hamiltonian acting on the $C_{3 v}$ symmetry defect can be separated into three irreducible components

$$
H_{s t r}=S_{z}^{\left(A_{1}\right)}+S_{x}^{\left(E_{x}\right)}+S_{y}^{\left(E_{y}\right)},
$$

where the symmetries of the terms are given in the parentheses. Axes $(x, y, z)$ are defined with $z$ parallel to the $\mathrm{NV}$ axis and $x$ lying in one of the three mirror planes, as shown in Figure 1(a). With only three components to the stress 
perturbation, it is clearly not possible to determine the full stress tensor from the properties of a single NV center. The key finding of this work is that measurement of the full tensor becomes possible if measurements are performed on three differently oriented centers experiencing the same strain field.

The three components of the perturbation hamiltonian in Eq. (1) can be written in terms of the components of the stress tensor $\sigma_{i j}$, grouped to share the corresponding symmetry. ${ }^{23}$ For simplicity, we consider here a stress perturbation that greatly exceeds the spin-orbit and spin-spin interaction energies, whereby the six ${ }^{3} \mathrm{E}$ states form two branches corresponding to orthogonal spatial orbitals $E_{x^{\prime}}$ and $E_{y^{\prime}}$, and the perturbation can be expressed as a secular matrix in the $\left(E_{x}, E_{y}\right)$ basis $^{23}$

$$
\left(\begin{array}{cc}
\alpha+\beta & \gamma \\
\gamma & \alpha-\beta
\end{array}\right)
$$

where $\quad \alpha=\left\langle E\left|S_{z}\right| E\right\rangle-\left\langle A\left|S_{z}\right| A\right\rangle, \quad \beta=\left\langle E\left|S_{x}\right| E\right\rangle, \quad$ and $\gamma=\left\langle E\left|S_{y}\right| E\right\rangle$. The eigenvalues and eigenvectors of this secular matrix give the transition energies and dipole orientations in the $(x, y)$ plane, respectively. The mean energy shift is $\alpha$ and the splitting is $\delta=2 \sqrt{\beta^{2}+\gamma^{2}}$, while the $E$ dipoles are rotated by an angle $\phi=1 / 2 \arctan (\gamma / \beta)$ relative to $x$ and $y$ (Figure 1(b)). For a [111] oriented NV center, this gives

$$
\begin{aligned}
& \alpha=A_{1}\left(\sigma_{x x}+\sigma_{y y}+\sigma_{z z}\right)+A_{2}\left(2 \sigma_{z z}-\sigma_{x x}-\sigma_{y y}\right), \\
& \beta=(B+C)\left(\sigma_{x x}-\sigma_{y y}\right)+\sqrt{2}(2 B-C) \sigma_{x z}, \\
& \gamma=-2(B+C) \sigma_{x y}+\sqrt{2}(2 B-C) \sigma_{y z},
\end{aligned}
$$

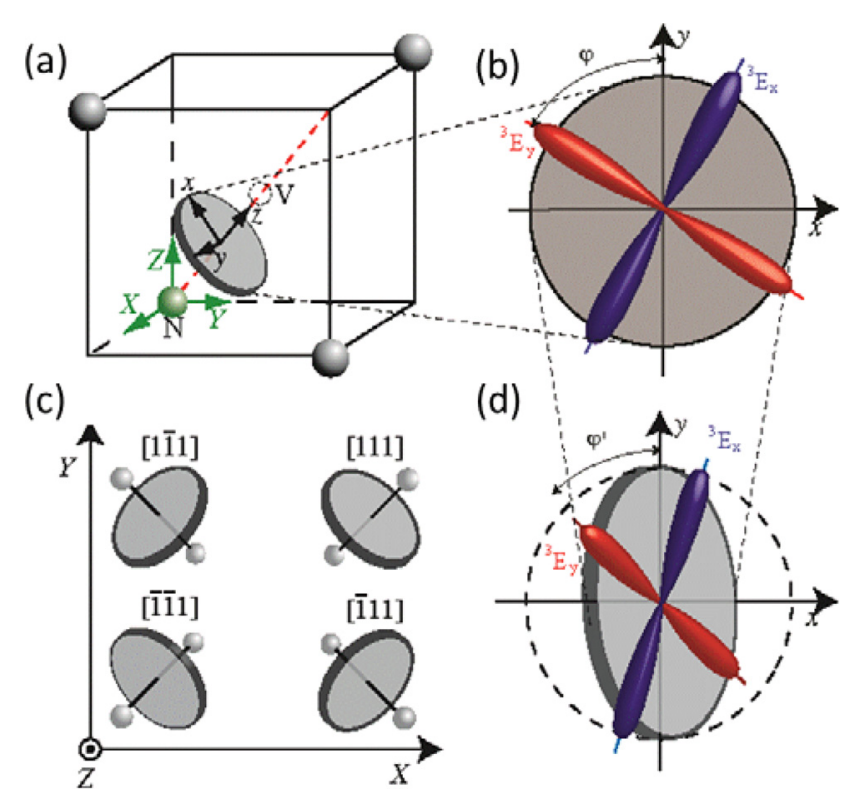

FIG. 1. (a) Schematic of the NV structure and definition of NV axes $(x, y, z)$ and crystal axes $(X, Y, Z)$ for an NV center with $z \|[111]$, whereby $x \|[\overline{1} 12]$ and $y \|[1 \overline{1} 0]$. The cube represents $\frac{1}{8}$ of the cubic unit cell of the diamond crystal. (b) Rotation of the $E_{x^{\prime}}$ and $E_{y^{\prime}}$ orbitals relative to NV axes $x$ and $y$ under a symmetry-breaking stress. $E_{x^{\prime}}$ is taken to be the higher energy orbital, consistent with Ref. 21. (c) Projection onto the (001) image plane $(X Y)$ of the four possible orientations of NVs relative to the crystal axes. (d) Projection of the $E_{x^{\prime}, y^{\prime}}$ orbitals in (b) onto the image plane. where parameters $A_{1}, A_{2}, B$, and $C$ have been determined as $1.47,-3.85,-1.04$, and $-1.69 \mathrm{meV} / \mathrm{GPa}$, respectively. ${ }^{24}$ Referred to the crystal $(X, Y, Z)$ axes, the equivalent equations are

$$
\begin{aligned}
& \alpha=A_{1}\left(\sigma_{X X}+\sigma_{Y Y}+\sigma_{Z Z}\right)+2 A_{2}\left(\sigma_{Y Z}+\sigma_{Z X}+\sigma_{X Y}\right) \\
& \beta=B\left(2 \sigma_{Z Z}-\sigma_{X X}-\sigma_{Y Y}\right)+C\left(2 \sigma_{X Y}-\sigma_{Y Z}-\sigma_{Z X}\right) \\
& \gamma=\sqrt{3} B\left(\sigma_{X X}-\sigma_{Y Y}\right)+\sqrt{3} C\left(\sigma_{Y Z}-\sigma_{Z X}\right)
\end{aligned}
$$

The equations for an NV center with one of the three other orientations can now be obtained by performing successive $\frac{\pi}{2}$ rotations about $Z$. To obtain all six components of the tensor, it is necessary to measure $\alpha, \beta$, and $\gamma$ for three NV centers that experience the same stress field but which have different orientations in the lattice. These constitute an overdetermined set of nine linear equations with six unknown variables, from which a unique solution is obtained using the Moore-Penrose pseudoinverse method. ${ }^{25}$

To demonstrate this method for establishing the stress tensor, we selected a sample of monocrystalline diamond grown by plasma assisted chemical vapour deposition, ${ }^{26}$ which displays PL from isolated NV centers in a region of particularly high grown-in stress (Figure 2(a)). At a temperature of $77 \mathrm{~K}$, many of the zero phonon lines (ZPLs) reveal clear splittings of up to $0.8 \mathrm{~nm}$ or $2.4 \mathrm{meV}$ (Figure 2(b)). The growth direction is $Z \|$ [001], (also the viewing axis) such that the integrated emission of a given NV center is approximately 3 : 1 polarized, allowing easy discrimination between $\mathrm{NVs}$ with axis parallel to [111] or [ $\overline{1} \overline{1} 1]$ and those with axis parallel to [ $\overline{1} 11]$ or $[1 \overline{1} 1]$ (Figure $1(\mathrm{c})$ ). The ambiguity (effectively a mirror in $Z$ ) in identifying the orientation of a given NV center is not possible to resolve with this sample geometry without application of an additional perturbation, but presents only a minor limitation to the results. We note that a [111] viewing axis would remove this ambiguity. ${ }^{27}$

The two emission lines of the ZPL doublet are linearly polarized along the respective dipole axes. The measured projections of these polarizations onto the (001) image plane allow determination of the angle $\phi$ and thus we obtain $\gamma=\delta \sin (2 \phi)$ and $\beta=\delta \cos (2 \phi)$. As the $Z$-projections of individual NV centers are not known, we performed measurements on several proximal centers and looked for groupings of parameters to identify different defect orientations. An example of a group of seven NVs studied is that labeled 1 to 7 in Figure 2(a). Polar plots of the optical polarization from four of the centers are shown in Figure 2(c), and the corresponding $\alpha, \beta, \gamma$ values listed in Table I.

In two cases, pairs of NVs that are spatially close (2 and 3 and 6 and 7) give very similar values for all parameters measured. The conclusion is that these NVs are co-oriented, and are not affected substantially by local perturbations to the lattice. Of the $22 \mathrm{NVs}$ measured in total for this study, we found six examples of pairs of proximal NVs whose parameters agreed to better than $10 \%$, suggesting that for these NVs the assumption of a dominant long-range stress field is valid. Several NVs also revealed behaviour that was inconsistent with the assumption, presumably resulting from the presence of a strong local stress field due to a nearby defect such as a lattice vacancy, or an electric field due to a trapped charge. 


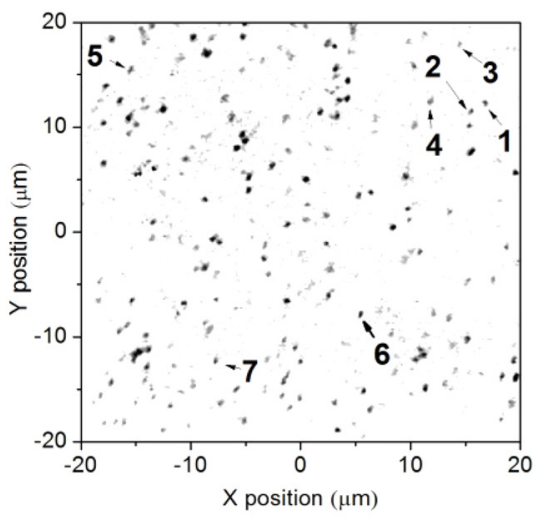

(a)

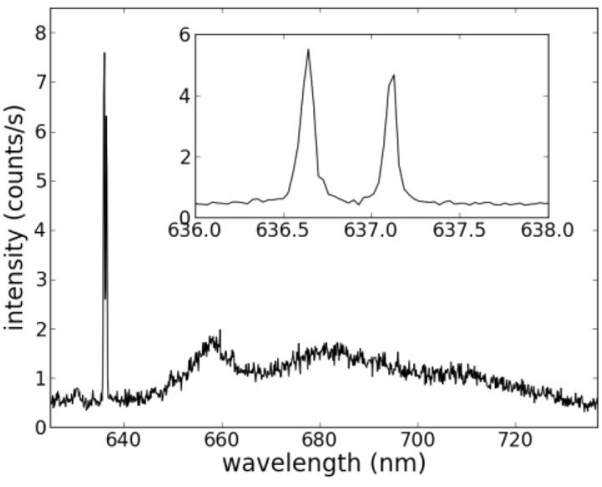

(b)

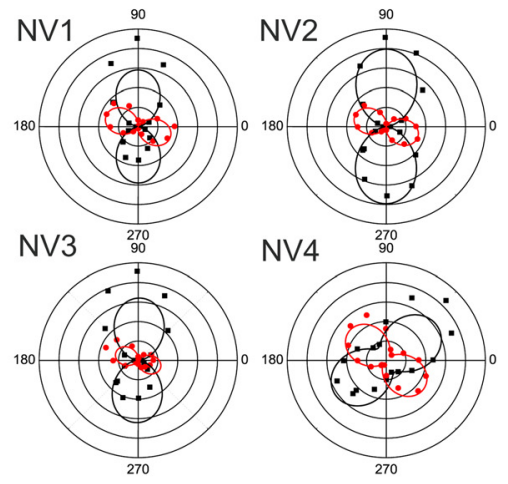

(c)

FIG. 2. Photoluminescence from individual NV centers in highly strained material at $77 \mathrm{~K}$ : (a) PL image of the sample area of interest with the seven NV centers used in this study labeled 1-7; (b) PL spectrum of a single NV center with a split zero phonon line (inset); (c) measured polarization data for NVs 1-4. The radius represents the emission intensity and the angle is that of a polarizing filter in the collection optics, $\phi_{p}$. The solid curves are fits to the function $I_{0}+$ $\Delta I \cos ^{2}\left(\phi_{p}+\phi^{\prime}\right)$ where $I_{0}, \Delta I$, and $\phi^{\prime}$ are fitting parameters.

We now use the data for NVs 1, (2 or 3), and 4 to calculate the local stress tensor in the top right of the image. Choosing the orientations NV1\| [1111], NV(2 or 3$) \|[1 \overline{1} 1]$, and NV4 $\|[111]$ yields the stress tensor

$$
\sigma=\left(\begin{array}{ccc}
290(5) & 90(0) & \pm 6(0) \\
90(0) & 758(0) & \pm 45(0) \\
\pm 6(0) & \pm 45(0) & -14(2)
\end{array}\right) \mathrm{MPa},
$$

where the figures presented are the mean values (standard deviations) for the tensor components calculated using the sets $\{\mathrm{NV} 1, \mathrm{NV} 2, \mathrm{NV} 4\}$ and $\{\mathrm{NV} 1, \mathrm{NV} 3, \mathrm{NV} 4\}$. The \pm values in $\sigma_{X Z}$ and $\sigma_{Y Z}$ indicate the effect of inverting $Z$ for all three centers. The tensor indicates a stress that is primarily biaxial in the (001) growth plane, with principal axes closely aligned to the [100] and [010] crystal axes. Reversing the choice of orientation for NV4 (only) creates a distinct configuration and results in the tensor

$$
\sigma^{\prime}=\left(\begin{array}{ccc}
259(1) & 98(2) & \pm 44(2) \\
98(2) & 758(4) & \pm 27(2) \\
\pm 44(2) & \pm 27(2) & -31(6)
\end{array}\right) \mathrm{MPa}
$$

The most significant differences between $\sigma$ and $\sigma^{\prime}$ are in the tensor components containing $Z$, corresponding to the $Z$ reflection of NV4.

We now use Eqs. (4) to recalculate $\alpha, \beta$, and $\gamma$ for each of the NV orientations to test of the self-consistency of the

TABLE I. Polarisation and energy shift data for seven NV centers labeled in Figure 2. Errors in each of the three parameters are estimated to be $0.1 \mathrm{meV}$.

\begin{tabular}{lcccr}
\hline \hline Defect & Angle & $\alpha(\mathrm{meV})$ & $\beta(\mathrm{meV})$ & $\gamma(\mathrm{meV})$ \\
\hline NV1 & 90 & 1.97 & 1.71 & -0.69 \\
NV2 & 90 & 2.36 & 1.39 & -0.56 \\
NV3 & 90 & 2.35 & 1.35 & -0.60 \\
NV4 & 0 & 1.32 & 0.50 & 1.38 \\
NV5 & 0 & 1.14 & 0.16 & 1.19 \\
NV6 & 0 & 1.69 & 0.30 & 1.09 \\
NV7 & 0 & 1.74 & 0.35 & 1.05 \\
\hline \hline
\end{tabular}

overdetermined set of equations. Tensor $\sigma$ yields the results presented in Table II which reproduce the parameters for NVs $1-4$ to within about $10 \%$. The orientations of NVs 5-7 appear most likely to be [111]; that their parameters differ somewhat from those of NV4 is attributed to longer range changes in the stress field.

Using a Young's modulus for diamond of $1200 \mathrm{GPa}$, the $\sim 10 \mathrm{MPa}$ sensitivity demonstrated here corresponds to a strain of $\sim 10^{-5}$. Much higher sensitivities can be achieved at lower temperatures, as the optical transition line widths reduce proportionally to $T^{5}$ due to the dynamic Jahn Teller effect. ${ }^{29}$ Photoluminescence excitation spectroscopy at liquid helium temperatures can be used to measure shifts about $100 \mathrm{MHz}$, increasing the strain sensitivity to $\sim 10^{-8}$. For determination of the full stress tensor under ambient conditions, it is equally possible to measure the perturbation to the electron spin state of the center using optically detected magnetic resonance. ${ }^{27}$ Dolde et al. ${ }^{30}$ have demonstrated sensitivity of this spin transition to electric fields of order $1 \mathrm{kV} \mathrm{cm}^{-1}$ $\mathrm{Hz}^{-\frac{1}{2}}$, suggesting that stresses of order $100 \mathrm{kPa} \mathrm{Hz}^{-\frac{1}{2}}$ could be measured in this way. With an NV electron spin dephasing time of order $1 \mathrm{~ms}$, this suggests a sensitivity of a few MPa may be achieved, equivalent to a strain of $\sim 10^{-6}$.

$\mathrm{NV}$ centers displaying behavior inconsistent with the effect of long range strain indicate the presence of an additional local strain field due to a nearby lattice defect. The magnitude of the strain field from a single substitutional nitrogen is approximately $6 \times 10^{-5} / r^{3}$ at a distance $r$ measured in nanometers, ${ }^{28}$ allowing the demonstrated sensitivity of $\sim 10^{-5}$ and maximum sensitivity of $\sim 10^{-8}$ to be converted to ranges of around $2 \mathrm{~nm}$ and $20 \mathrm{~nm}$, respectively.

TABLE II. Recalculated energy perturbations for the four NV orientations using stress tensor $\sigma$.

\begin{tabular}{lrccr}
\hline \hline NV axis & angle & $\alpha(\mathrm{meV})$ & $\beta(\mathrm{meV})$ & $\gamma(\mathrm{meV})$ \\
\hline$[111]$ & 0 & 1.22 & 0.73 & 0.96 \\
{$[1 \overline{1} 1]$} & 90 & 2.51 & 1.36 & -0.99 \\
{$[\overline{1} \overline{1} 1]$} & 0 & 0.43 & 0.90 & 0.73 \\
{$[\overline{1} 11]$} & 90 & 1.91 & 1.49 & -0.70 \\
\hline \hline
\end{tabular}


Such information may be useful where coupled systems, or conversely where NV centers free of influence from nearby defects, are required.

In summary, we have shown that the full stress tensor in diamond can be measured using fluorescence from NV centers. The method will be useful for developing diamondbased quantum devices that require detailed knowledge of the local crystal environment and has broader potential for use in high sensitivity nanoscale stress analysis in suitable materials.

We would like to thank A. J. Wilkinson for helpful discussions regarding stress and strain measurement techniques. The work was funded by the United Kingdom Engineering and Physical Sciences Research Council through the Quantum Information Processing Interdisciplinary Research Collaboration (Grant Reference No. GR/S822176/01). F.G. and J.M.S. acknowledge additional support from Hewlett Packard Ltd.

${ }^{1}$ B. E. Kane, Nature 393, 133 (1998).

${ }^{2}$ G. H. Loechelt, N. G. Cave, and J. Menéndez, J. Appl. Phys 86, 6164 (1999).

${ }^{3}$ Y. Tang, D. H. Rich, E. H. Lingunis, and N. M. Haegel, J. Appl. Phys 76, 3032 (1994).

${ }^{4}$ A. S. Voloshin and C. P. Burger, Exp. Mech. 23, 304 (1983).

${ }^{5}$ A. J. Schwartz, M. Kumar, B. L. Adams, and D. Field, Electron Backscatter Diffraction in Materials Science (Springer, New York, 2009).

${ }^{6}$ J. F. Nye, Physical Properties of Crystals (Oxford University Press, 1957).

${ }^{7}$ E. Rittweger, K. Y. Han, S. E. Irvine, C. Eggeling, and S. W. Hell, Nat. Photonics 3, 144 (2009).

${ }^{8}$ C. Kurtsiefer, S. Mayer, P. Zarda, and H. Weinfurter, Phys. Rev. Lett. 85, 290 (2000).

${ }^{9}$ N. B. Manson, J. P. Harrison, and M. J. Sellars, Phys. Rev. B 74, 104303 (2006).

${ }^{10}$ A. Batalov, V. Jacques, F. Kaiser, P. Siyushev, P. Neumann, L. J. Rogers, R. L. McMurtrie, N. B. Manson, F. Jelezko, and J. Wrachtrup, Phys. Rev. Lett. 102, 195506 (2009).

${ }^{11}$ M. W. Doherty, N. B. Manson, P. Delaney, and L. C. L. Hollenberg, New J. Phys. 13, 025019 (2011).
${ }^{12}$ J. R. Maze, A. Gali, E. Togan, Y. Chu, A. Trifonov, E. Kaxiras, and M. D. Lukin, New J. Phys. 13, 025025 (2011).

${ }^{13}$ M. W. Doherty, F. Dolde, H. Fedder, F. Jelezko, J. Wrachtrup, N. B. Manson, and L. C. L. Hollenberg, Phys. Rev. B 85, 205203 (2012).

${ }^{14}$ F. Jelezko, T. Gaebel, I. Popa, A. Gruber, and J. Wrachtrup, Phys. Rev. Lett. 92, 076401 (2004).

${ }^{15}$ M. V. Gurudev Dutt, L. Childress, L. Jiang, E. Togan, J. Maze, F. Jelezko, A. S. Zibrov, P. R. Hemmer, and M. D. Lukin, Science 316, 1312 (2007).

${ }^{16}$ P. Mauer, G. Kucsko, L. Jiang, F. Pastawski, D. J. Twitchen, J. I. Cirac, and M. D. Lukin, Science 336, 1283 (2012).

${ }^{17}$ G. D. Fuchs, V. V. Dobrovitski, D. M. Toyli, F. J. Heremans, and D. D. Awschalom, Science 326, 1520 (2009).

${ }^{18}$ C. Santori, P. Tamarat, P. Neumann, J. Wrachtrup, D. Fattal, R. G. Beausoleil, J. Rabeau, P. Olivero, A. D. Greentree, S. Prawer, F. Jelezko, and P. Hemmer, Phys. Rev. Lett. 97, 247401 (2006).

${ }^{19}$ E. Togan, Y. Chu, A. S. Trifonov, L. Jiang, J. Maze, L. Childress, M. V. G. Dutt, A. S. Srensen, P. R. Hemmer, A. S. Zibrov, and M. D. Lukin, Nature 466, 730 (2010).

${ }^{20}$ C. A. Coulson and M. J. Kearsley, Proc. R. Soc., London, Ser. A 241, 433 (1957).

${ }^{21}$ P. Tamarat, T. Gaebel, J. R. Rabeau, M. Khan, A. D. Greentree, H. Wilson, L. C. L. Hollenberg, S. Prawer, P. Hemmer, F. Jelezko, and J. Wrachtrup, Phys. Rev. Lett. 97, 083002 (2006).

${ }^{22}$ J. F. Cornwell, Group Theory in Physics: An Introduction (Academic, London, 1997).

${ }^{23}$ A. E. Hughes and W. A. Runciman, Proc. Phys. Soc. 90, 827 (1967).

${ }^{24}$ G. Davies and M. F. Hamer, Proc. R. Soc., London, Ser. A 348, 285 (1976).

${ }^{25}$ R. Penrose and J. A. Todd, Math. Proc. Cambridge Philos. Soc. 52, 17 (1956).

${ }^{26}$ R. S. Balmer, J. R. Brandon, S. L. Clewes, H. K. Dhillon, J. M. Dodson, I. Friel, P. N. Inglis, T. D. Madgwick, M. L. Markham, T. P. Mollart, N. Perkins, G. A. Scarsbrook, D. J. Twitchen, A. J. Whitehead, J. J. Wilman, and S. M. Woollard, J. Phys.: Condens. Matter 21, 364221 (2009).

${ }^{27}$ See supplementary material at http://dx.doi.org/10.1063/1.4819834 for further details of the sample, alternate viewing geometries, and a description of how the technique may be employed at ambient temperatures using spin measurements.

${ }^{28}$ G. Davies, J. Phys. C 3, 2474 (1970).

${ }^{29}$ K. - M. C. Fu, C. Santori, P. E. Barclay, L. J. Rogers, N. B. Manson, and R. G. Beausoleil, Phys. Rev. Lett. 103, 256404 (2009).

${ }^{30}$ F. Dolde, H. Fedder, M. W. Doherty, T. Nöbauer, F. Rempp, G. Balasubramanian, T. Wolf, F. Reinhard, L. C. L. Hollenberg, F. Jelezko, and J. Wrachtrup, Nat. Phys. 7, 459 (2011). 